\title{
Modeling the rapid spherical compression of isotropic turbulence
}

\author{
Gary N. Coleman and Nagi N. Mansour \\ NASA Ames Research Center, Moffett Field, California 94035
}

(Received 5 February 1991; accepted 1 May 1991)

\begin{abstract}
Rapid distortion analysis is used to modify the form of the closure model for the dissipation rate of the turbulent kinetic energy. The modification is such that the evolution of the dissipation rate during a rapid compression is predicted exactly; good agreement between the model prediction and direct simulation data is obtained. Previous closure proposals fail to properly predict the rapid compression case. The reason for the difference between the present and previous models is traced to the fact that previous workers neglected variations of kinematic viscosity.
\end{abstract}

\section{INTRODUCTION}

The ability to correctly predict a wide range of flowsincluding those associated with internal combustion engines, hypersonic flight, supersonic combustion, and astrophysical phenomena-depends in large part on how well one is able to model the behavior of compressible turbulence. Unfortunately, the accuracy of current compressible turbulence models is often unacceptable (see, for example, Vuong

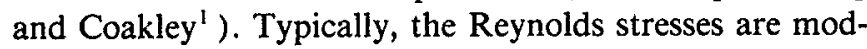
eled using an eddy viscosity based on the turbulent kinetic energy $k$ and its dissipation rate $\epsilon$, and transport equations for $k$ and $\epsilon$ must be closed. The objective of this study is to improve the closure model for the $\epsilon$ equation in predicting flows under bulk compression. There is an acute need for compressible models that are reliable at various Reynolds numbers, Mach numbers, and strain rates. By requiring a model to be exact for one limiting case, it is hoped that it will represent the physics better for all flows.

Our approach is to compare model predictions with results from direct numerical simulation (DNS) of homogeneous compressible turbulence at low fluctuation Mach numbers (velocity fluctuations small compared to the local sound speed) subjected to rapid three-dimensional (spherical, isotropic) compression. An advantage of considering this type of straining is that it allows rapid distortion theory (RDT) to be used to rigorously test and modify the models. In particular, RDT determines the form of the model equation for the transport of the rate of turbulent kinetic energy dissipation required in the rapid compression limit. This study is therefore relevant to the fields of two-equation and second-order modeling.

The use of RDT for spherically compressed flows to derive a closure to the $\epsilon$ equation was introduced by Reynolds. $^{2}$ Morel and Mansour ${ }^{3}$ extended Reynolds' work to admit other types of compressions, including those most relevant to internal combustion engines (one-dimensional and cylindrical-radial compressions). Recently, the Morel and Mansour model has been included in Mao's ${ }^{4}$ numerical study of flows in internal combustion engines. Although the imposed compression rates were not rapid (which implies that the model could not bc exact), fairly good agreement with experimental data was reported. A direct comparison of RDT with measurements of turbulence within internal combustion engines has been made by Hoult and Wong, ${ }^{5}$ who concluded that the theory can duplicate the experimental data reasonably well. These studies provide examples of the way in which RDT can be applied to problems of practical interest.

Spherical compression of turbulence at low Mach number has also been studied by Wu et al., ${ }^{6}$ who have also compared model predictions with DNS data. One of their primary conclusions was that a third model equation-in addition to the transport equations for turbulent kinetic energy and its dissipation rate-which represents the evolution of a turbulent time scale, was required to reproduce the DNS results. While the third equation may be necessary for general flow conditions, we find that a properly modified $\epsilon$ equation is sufficient to capture the behavior of $k$ and $\epsilon$ when the compression rate is rapid compared to the turbulence time scales.

The purpose of this paper is to reexamine and generalize the previous rapid distortion analyses of Reynolds ${ }^{2}$ and Morel and Mansour, ${ }^{3}$ and to explore the implications for closure of the $\epsilon$ equation. We find that the validity of the previous studies is limited, since they neglect the variation of kinematic viscosity during the compression, and make use of an equilibrium relationship that is not appropriate during rapid strain. In what follows, RDT is used to determine the modification to the $\epsilon$ equation required to follow the low Mach number turbulence through a rapid spherical compression. Predictions given by the various models are compared with results of direct simulations of homogeneous compressible turbulence. The physical implications of the suggested model and the likely effects of Mach and Reynolds numbers are discussed.

\section{RAPID COMPRESSION AT LOW FLUCTUATION MACH NUMBER}

We consider homogeneous compressible turbulence at small fluctuation Mach number. The turbulent velocity is effectively divergence-free and the density is uniform in space but may vary with time. The flow cxperiences a mean strain given by

$$
U_{i}=U_{i, j}(t) x_{j},
$$

with

$$
U_{i, j}(t)=D(t) \delta_{i j} / 3,
$$


where $U_{i}$ is the mean (ensemble averaged) velocity and $D$ the mean divergence; here $D<0$, denoting a mean compression. The class of strains defined by (1) and (2) will maintain the homogeneity of a flow that is initially homogeneous, for any straining history $D(t)$, since the density depends only on time. ${ }^{7}$ If $\rho$ was not uniform in space, Blaisdell et al. ${ }^{7}$ have shown that the mean dilatation introduces inhomogeneity unless the compression follows a specific time history,

$$
D(t)=D(0) /[1+D(0) t / 3]
$$

(this is the strain which causes the length of a fluid element to change at a constant rate; it is used in the simulations discussed below).

For the low Mach number, isotropic compression case, the equations that describe the evolution of the turbulent kinetic energy, $k=\frac{1}{2} \overline{u_{i} u_{i}}$, and the enstrophy, $\omega^{2}=\overline{\omega_{i} \omega_{i}}=\epsilon / v$, become

$$
\frac{d k}{d t}=-\frac{2}{3} k D-\epsilon
$$

and

$$
\frac{d \omega^{2}}{d t}=-\frac{4}{3} D \omega^{2}+2 \overline{\omega_{i} \omega_{j} u_{i j}}-2 v \overline{\omega_{i, j} \omega_{i, j}}
$$

During a rapid compression, the dominant terms on the right-hand side of (4) and (5) are those which contain $D$, since the time scale of the mean compression, $\left|D^{-1}\right|$, is much smaller than the time scales associated with the other source terms: for Eq. (4), this amounts to making the classic RDT assumption, $|D k / \epsilon| \gg 1\left(\right.$ Hunt $\left.^{8}\right)$, while in (5) we are assuming that $\left|D \lambda^{2} / \nu\right| \gg 1$, where $\lambda$ is a Taylor microscale [which is another way of writing $|D k / \epsilon| \gg 1$; see Eq. (19)] and also $\left|D / \sqrt{\omega^{2}}\right| \gg 1$. Since $\left|D / \sqrt{\omega^{2}}\right|=|D k / \epsilon| \operatorname{Re}_{T}^{-1 / 2}$, where $\operatorname{Re}_{T}$ is the turbulent Reynolds number $k^{2} / \epsilon v$, we observe that the RDT regime is defined by both the strain rate and the Reynolds number of the flow; as $\mathrm{Re}_{T}$ increases, the $D$ required to satisfy the RDT constraints also increases.

A rapid spherical compression therefore leads to

$$
\frac{d k}{d t}=-\frac{2}{3} D k
$$

and

$$
\frac{d \omega^{2}}{d t}=-\frac{4}{3} D \omega^{2}
$$

From (6) and (7) we have

$$
k(t)=k(0) \exp \left(-2 D^{*}(t) / 3\right)
$$

and

$$
\omega^{2}(t)=\omega^{2}(0) \exp \left(-4 D^{*}(t) / 3\right),
$$

where $D^{*}(t)=\int_{0}^{t} D(\sigma) d \sigma$. It can be shown, using the results of this and later sections [Eqs. (8), (9), (15), (16), and (22)], that as $t \rightarrow \infty,|D k / \epsilon|$ increases during a rapid compression and decreases during an expansion. For the straining given by (3), this holds for almost all gases of interest [those which demonstrate viscosity variations such that the exponent in Eq. (15), $n<2 / 3(\gamma-1)]$. The other RDT constraint, $\left|D / \sqrt{\omega^{2}}\right|$, exhibits the opposite behavior and grows only during an expansion. Thus, (6)-(9) are in gen- eral only valid for times short compared to $\left|D^{-1}\right|$. However, because compressions can continue only for a finite time before the flow volume collapses to zero [under the Eq. (3) straining, this occurs at $t=-3 / D(0)]$, for the $D(t)<0$ case considered here, the RDT results above may apply for a large fraction of the total straining history.

\section{MODELING RAPID COMPRESSION}

We now use the rapid distortion analysis of the previous section to evaluate compressible turbulence models for low Mach number flows. In the case of spherical compression, the Reynolds stress and kinetic energy transport equations are consistent with RDT, since they are exact (i.e., involve no modeling). Our focus must therefore be on the $\epsilon$ equation, which must be modeled. A form appropriate for low Mach number compressible cases is (Gosman and Watkins, ${ }^{9}$ Reynolds, ${ }^{2}$ Morel and Mansour; ${ }^{3}$ see also El Tahry ${ }^{10}$ for an alternative form, discussed below)

$$
\begin{aligned}
\rho\left(\frac{\partial \epsilon}{\partial t}+U_{j} \frac{\partial \epsilon}{\partial x_{j}}\right)= & \frac{\epsilon}{k}\left(C_{1} P-C_{2} \rho \epsilon\right) \\
& +\left(1-C_{3}\right) \rho \epsilon D+\text { diffusion. }
\end{aligned}
$$

The production of $k, P=-\rho \overline{u_{i} u_{j}} S_{i j}$, is modeled using the eddy viscosity assumption as

$$
P=2 C_{\mu} \rho\left(k^{2} / \epsilon\right)\left(S_{i j} S_{i j}-D^{2} / 3\right)-2 \rho k D / 3,
$$

where $S_{i j}=\frac{1}{2}\left(U_{i, j}+U_{j, i}\right)$. Popular values for the model constants used in (10) and (11) are $C_{\mu}=0.09, C_{1}=1.44$, and $C_{2}=1.92$ (Patel et al. ${ }^{11}$ ).

The coefficient $C_{3}$ has been included in (10) so that the $\epsilon$ equation can be made exact for rapid spherical compressions (Reynolds ${ }^{2}$ ); our objective is to find the appropriate value for $C_{3}$. We first apply the $\epsilon$ equation to the (not necessarily rapid) spherical mean compression of homogeneous turbulence. The production of $k$ is then $P=-2 \rho k D / 3$, the diffusion terms vanish, and (10) reduces to

$$
\frac{d \epsilon}{d t}=\left(-\frac{2}{3} C_{1}+1-C_{3}\right) D \epsilon-C_{2} \frac{\epsilon^{2}}{k} \text {. }
$$

We now use the fact that for a homogeneous low Mach number flow $\epsilon \equiv v \omega \omega^{2}$. (Beyond the low Mach number regime, $\epsilon$ also contains the "extra dissipation" associated with smallscale dilatation fiuctuations, which can be significant in some situations. See Sarkar et al., ${ }^{12}$ Zeman, ${ }^{13}$ Aupoix et $a l .,^{14}$ Blaisdell et al., ${ }^{7}$ or Speziale and Sarkar ${ }^{15}$ for discussions of how this and other high fluctuation Mach number effects might be modeled.) Equation (12) thus becomes

$$
\frac{d \omega^{2}}{d t}=\left[\left(-\frac{2}{3} C_{1}+1-C_{3}\right) D-\frac{1}{v} \frac{d v}{d t}-C_{2} \frac{v \omega^{2}}{k}\right] \omega^{2} \text {. }
$$

Equation (13) models the exact enstrophy equation (5). For rapid compression, $|D k / \epsilon| \geqslant 1$ and $(1 / v) d v / d t$ is of order $D$ [see (16) below], so the terms in (12) and (13) containing $C_{2}$ are insignificant. Comparing (13) with (7), we see that for the $\epsilon$ equation (10) to be exact during a rapid spherical compression, the model constants must satisfy 


$$
\left(-\frac{2}{3} C_{1}+1-C_{3}\right) D-\frac{1}{v} \frac{d v}{d t}=-\frac{4}{3} D
$$

or

$$
C_{3}=\frac{\left[7-(3 / D v)(d v / d t)-2 C_{1}\right]}{3} .
$$

For an ideal gas with constant specific heats and temperature-dependent viscosity given by

$$
\mu(T)=\mu\left(T_{0}\right)\left(T / T_{0}\right)^{n},
$$

we have (assuming an isentropic compression)

$$
\frac{1}{D v} \frac{d v}{d t}=1-n(\gamma-1)
$$

so that (14) can be written

$$
C_{3}=\left[4+3 n(\gamma-1)-2 C_{1}\right] / 3 \text {, }
$$

where $\gamma$ is the ratio of specific heats.

This expression for $C_{3}$ is different from that proposed by Reynolds $^{2}$ and Morel and Mansour, ${ }^{3}$

$$
C_{3}=\left(7-2 C_{1}\right) / 3 \text {. }
$$

The origin of the difference and a comparison of the resulting dissipation predictions will be discussed below.

\section{COMPARISON WITH DIRECT SIMULATION RESULTS}

Now that we have proposed a new formulation for the $\epsilon$ equation, we turn our attention to its verification. The basis for evaluating the models associated with the new and previous expressions for $C_{3}$, Eqs. (14) and (18), respectively, will be DNS data. In a DNS, turbulent flow fields are generated by numerically solving the Navier-Stokes equations, resolving all relevant scales of motion, without resorting to turbulence models. Although DNS results are limited to low Reynolds numbers, they contain no modeling errors-and can therefore be viewed as "experimental data" for our purposes. Since viscous effects are negligible during a rapid compression (i.e., $|D k / \epsilon|=\left|D \lambda^{2} / v\right|>1$ ), the fact that the DNS data are at low Reynolds number should not be a disadvantage here.

One of the Wu et al. ${ }^{6}$ simulations of low Mach number, spherically compressed turbulence (their Case SQF) had a sufficiently high compression rate so that the results can be used for the model evaluation. In the Wu et al. simulations the fluctuation velocity was solenoidal, and the incompressible form of the transport equations was used, so the fluctuation Mach number, $\boldsymbol{M}_{\mathrm{rms}}$, was identically zero. The imposed compression followed (3), with $(D k / \epsilon)_{0}=-70.5$ (a zero subscript will be used throughout to denote an initial value). The initial production of $k$ was thus $2(70.5) / 3=47$ times larger than the initial dissipation rate of $k$, consistent with rapid compression. The other parameter defining this flow was the initial turbulent Reynolds number: $\left(\operatorname{Re}_{T}\right)_{0}=\left(k^{2} / \epsilon v\right)_{0}=5.1$ for Case SQF. This Reynolds number, which we shall see is high enough so that viscous effects are not important, implies that $\left(D / \sqrt{\omega^{2}}\right)_{0}$ $=\left(\operatorname{Re}_{T}\right)_{0}^{-1 / 2}(D k / \epsilon)_{0}=-31.2$. Since $|D k / \epsilon|_{0}$ and
$\left|D / \sqrt{\omega^{2}}\right|_{\text {o }}$ were $\gg 1$, both measures of rapid distortion were satisfied.

We have also run an independent simulation of this case, using the fully compressible Navier-Stokes code of Blaisdell et al. with initial $M_{\mathrm{rms}}=0.04,(D k / \epsilon)_{0}=-70.5$, $\mathbf{R e}_{T}=5.1, \gamma=1.4$, and viscosity exponent $n=0.75$ (other run parameters are listed in the Appendix).

The dissipation histories of the computations are presented in Fig. 1; very good agreement is observed between the Wu et al. results (symbols) and the present simulation data (dotted curve) over the time interval shown, $t \leqslant 0.5 / S_{0}=0.5 /[-D(0) / 3]$. At $S_{0} t=0.5$, the compression has progressed halfway to the "singularity time," the point at which the density becomes infinite. Over this range, $|D k / \epsilon|$ increases from 70.5 at $S_{0} t=0$ to 151 at $S_{0} t=0.5$, while $\left|D / \sqrt{\omega^{2}}\right|$ falls from 30.2 to 15.1 . The latter is predicted by (3) and (9), which imply $\left|D / \sqrt{\omega^{2}}\right|_{0} /\left|D / \sqrt{\omega^{2}}\right|=1-S_{0} t$.

Since both RDT parameters remain large for the time considered, the rapid distortion approximations should accurately represent the Fig. 1 histories. In fact, from the above expression we have that for $S_{0} t<0.8,\left|D / \sqrt{\omega^{2}}\right|>5$, so the RDT results are apt to be valid at times later than those presented in Fig. 1.

Also shown in Fig. 1 are the predictions given by (12) when $C_{3}$ is defined by (18) (dashed curve) and (14) (chaindotted). The accuracy of the latter, and inaccuracy of the former, is striking. The slight difference between the DNS results and the Eq. (14) prediction is caused by the use of a nonzero "decay constant" $\left(C_{2}=1.92\right)$ in (12). When $C_{2}$ is set to zero, the solid curve shown in Fig. 1 is obtained, which agrees even better with the DNS data. The model comparison thus seems to strongly validate the approach and results of the previous section.

\section{DISCUSSION AND CONCLUSIONS}

An important step in each of the previous authors' derivation of Eq. (18) was a seemingly sound assumption concerning the behavior of the flow during a rapid compression. For example, Reynolds assumed that the bulk angular mo-

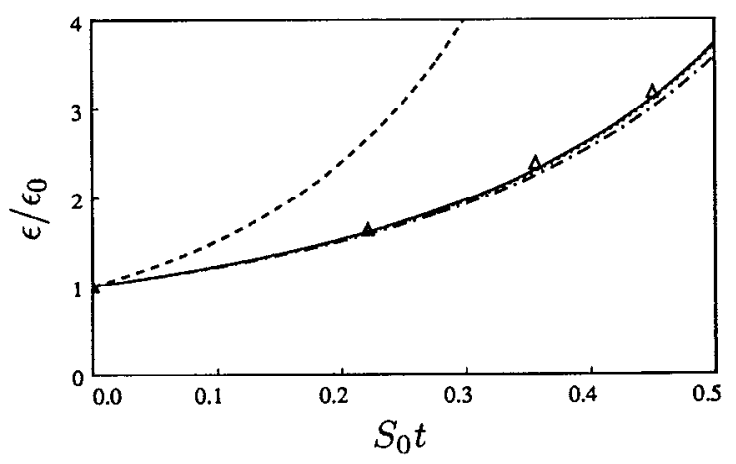

FIG. 1. Evolution of turbulent kinetic energy dissipation during a rapid spherical compression, $(D k / \epsilon)_{0}=-70.5,\left(k^{2} / \epsilon v\right)_{0}=5.1, M_{\mathrm{rms}} \approx 0 ; \Delta$, the Wu et al. ${ }^{6}$ DNS Case SQF, $M_{\text {rms }}=0 ; \cdots$, present DNS, $M_{\text {rms }}=0.04$; ----, prediction given by (12), using (18) for $C_{3}$, with $C_{2}=1.92 ;-^{-}-$, prediction given by (12), using (14) for $C_{3}$, with $C_{2}=1.92$; — , prediction given by (12), using (14) for $C_{3}$, with $C_{2}=0 ; S_{0}=-D(0) / 3$. 
mentum is conserved, while Morel and Mansour assumed that a turbulent length scale varies in direct proportion to the imposed mean compression. Since we now advocate a different $C_{3}$, it is reasonable to determine what physical constraint is implied by (14), and how it is related to the previous "physical" pictures of the effect of mean compression on the flow. To do this, we follow an analysis like that of Morel and Mansour, beginning with a Taylor microscale, $\lambda$, defined as

$$
\lambda^{2}=k / \omega^{2} \text {. }
$$

Differentiating (19) leads to

$$
\frac{1}{\lambda^{2}} \frac{d \lambda^{2}}{d t}=\frac{1}{k} \frac{d k}{d t}-\frac{1}{\omega^{2}} \frac{d \omega^{2}}{d t} .
$$

Substituting the RDT relations (6) and (7) into (20) gives

$$
\frac{1}{\lambda^{2}} \frac{d \lambda^{2}}{d t}=\frac{2}{3} D
$$

Upon integrating (21), and using the continuity equation,

$$
\frac{d \rho}{d t}=-\rho D
$$

we find that the constraint satisfied during a rapid compression is

$$
\rho \lambda^{3}=\text { const. }
$$

During a rapid compression the Taylor microscale and the velocity correlation integral scale $l_{*}$ remain proportional to each other, since the "shape" of the energy spectrum does not change (Wu et al. ${ }^{6}$ ), so we can recast (23) as

$$
\rho l^{3}=\text { const. }
$$

Equation (24) is the physical constraint associated with (14). The above expression implies that during a rapid compression, the "size of the large eddies" scales on the size of the flow domain - a consequence of the fact that during a rapid spherical compression, any length scale defined using the energy spectrum alone will scale directly with the total strain. This constraint seems plausible, and is in fact identical to that invoked by Morel and Mansour to recommend (18), with the critical exception that they took "eddy size" to be the dissipation length scale, $L_{\epsilon}=C_{\mu}^{3 / 4} k^{3 / 2} / \epsilon$. Reynolds also interpreted $L_{\epsilon}$ to be the size of the large eddies, and postulated that during the compression $k^{2} / \epsilon=$ const (consistent with conservation of angular momentum of the turbulence). The reason that both of these assumptions led to (18) - an expression for $C_{3}$ which has been found to be inaccurate-is that in order to relate $l_{*}$ to $L_{\varepsilon}$, one needs to assume either equilibrium between the large and small scales, or that $v$ remains constant. During a rapid compression, the turbulence is not in equilibrium (since there is no turbulent transfer of energy between scales), and the size of the large eddies will not in general be given by $L_{\epsilon}$. From (8), (9), and (19), we have $L_{\epsilon} / \lambda \sim L_{\epsilon} / l_{*} \sim 1 / v$, which demonstrates that $l_{*}$ will scale with $L_{\epsilon}$ only when $v$ remains constant during the compression (so that $\epsilon$ remains proportional to the enstrophy). This is also borne out by comparing Eqs. (14) and (18), where we see that when $d v / d t=0$, the two expressions for $C_{3}$ are identical. For $v$ to remain constant would require the dynamic viscosity to vary in exactly the same manner as the density; for an isentropic, constant $\gamma$ ideal gas, the viscos- ity law exponent in (15) would have to be $n=1 /(\gamma-1)$, which is in general not the case.

Wu et al. ${ }^{6}$ proposed a three equation scheme that models the evolution of $k, \epsilon$, and a turbulent lime scale, to correctly capture the $\epsilon$ history during a rapid three-dimensional compression. It is possible that three equations are needed for general compressible flows, but we have shown that by modifying the $\epsilon$ equation, rapid isotropic compression may be correctly represented using the $k-\epsilon$ set. Identical results could be obtained using a $k$-enstrophy approach. Our findings are relevant for both two-equation and second-order compressible turbulence models, and Eq. (14) should have a place in both fields.

It is noteworthy that our conclusions are independent of Reynolds number, since an inviscid analysis was used to derive a model constant that matches low Reynolds number DNS data. When compressibility effects are significant, $M_{\text {rms }}$ is not small, and terms such as the pressure-dilatation correlation, and the dilatation dissipation, which were neglected in our analysis, will not necessarily be small. However, since the mean production terms will still dominate, the low $M_{\text {rms }}$ results should still be approximately valid.

The importance of incorporating the compression-induced variation of $v$ in the dissipation equation that we have found above has been previously pointed out by El Tahry. ${ }^{10}$ His recommended form of the $\epsilon$ equation may be obtained by neglecting $C_{1}$, but keeping the $d v / d t$ term, in (14); because $C_{1}$ is neglected, the model advocated by El Tahry will not be exact for the case of rapid spherical compression.

By requiring a model to correctly predict a rapidly strained flow, our goal has been to improve its accuracy for other situations. The degree to which this goal is met, as well as the performance of El Tahry's, Reynolds', and Morel and Mansour's models when applied to nonrapid, arbitrarily strained cases, will be addressed in a future paper.

\section{ACKNOWLEDGMENTS}

The authors are grateful to G. Blaisdell, R. Rogallo, and O. Zeman for their contributions to this study.

GNC has been supported during this project by the National Research Council. Computer resources have been supplied by the Advanced Computational Facility and the National Aerodynamic Simulator at NASA Ames Research Center.

\section{APPENDIX: SIMULATION PARAMETERS}

The results shown in Fig. 1 were produced using the homogeneous compressible turbulence code of Blaisdell et al., ${ }^{7}$ employing $96^{3}$ grid points. This program computes numerical solutions of the compressible Navier-Stokes equations-for strains of various types-utilizing a spectral (Fourier) spatial discretization and a finite difference time advance scheme. An ideal gas with constant specific heats, and temperature-dependent viscosity given by (15), is assumed.

Initial conditions for the simulation were obtained by specifying isotropic velocity, temperature, and density fields 
TABLE I. DNS fluid and initial condition parameters.

\begin{aligned} & \hline Ratio of specific heats: $\gamma=1.4 \\ &$ Viscosity exponent [see Eq. (14)]: $n=0.75 \\ &$ Turbulent Reynolds number: $k^{2} / \epsilon v=5.1 \\ &$ Fluctuation Mach number: $M_{\mathrm{rms}}=0.04 \\ &$ Ratio of nonsolenoidal to total \\ & turbulent kinetic energy: $\chi=1.7 \times 10^{-4} \\ &$ Density fluctuation ratio; \\ & rms to volume average: $\rho_{\mathrm{rms}}=9.3 \times 10^{-4} \\ &$ Temperature fluctuation ratio; \\ & rms to average (absolute): $T_{\text {rms }}=3.4 \times 10^{-4} \\ &$\hline\end{aligned}

given by "top-hat" spectra, ${ }^{7}$ and then allowing the flow to decay without imposed strain, to a mature turbulent state which is determined by the velocity derivative skewness attaining a value $\simeq-0.4$. At this state, mean compression was imposed upon a mature flow with the characteristics listed in Table I; these values were chosen to duplicate those found in Case SQF of Wu et al. ${ }^{6}$ To match the Case SQF compression, the straining given by $(D k / \epsilon)_{0}=-70.5$, with $D(t)=D(0) /[1+D(0) t / 3]$, was prescribed. Further details concerning the solution procedure and numerical method may be found in Blaisdell et al. ${ }^{7}$

'S. T. Vuong and T. J. Coakley, AIAA Paper No. 87-0286, 1987.

${ }^{2}$ W. C. Reynolds, in Combustion Modeling in Reciprocating Engines, edited by J. N. Mattavi and C. A. Amann (Plenum, New York, 1980).

${ }^{3}$ T. Morel and N. N. Mansour, SAE Paper No. 820040, 1982.

${ }^{4}$ Y. Mao, Ph.D. dissertation, Ecole Centrale de Lyon, 1990.

${ }^{5}$ D. P. Hoult and V. W. Wong, in Ref. 2.

${ }^{6}$ C.-T. Wu, J. H. Ferziger, and D. R. Chapman, Stanford University, Department of Mechanical Engineering Report No. TF-21, 1985.

${ }^{7}$ G. A. Blaisdell, N. N. Mansour, and W. C. Rcynolds, Stanford University, Department of Mechanical Engineering Report No. TF-50, 1991.

${ }^{8}$ J. C. R. Hunt, Fluid Dyn. Trans. 9, 121 (1978).

${ }^{9}$ A. D. Gosman and A. P. Watkins, Proceedings of the First Symposium on Turbulent Shear Flows (Pennsylvania State University, University Park, PA, 1977), p. 5.23.

${ }^{10}$ S. H. El Tahry, J. Energy 7, 345 (1983).

${ }^{11}$ V. C. Patel, W. Rodi, and G. Scheuerer, AIAA J. 23, 1308 (1985).

${ }^{12}$ S. Sarkar, G. Erlebacher, M. Y. Hussaini, and H. O. Kreiss, ICASE Report No. 89-79, 1989.

${ }^{13}$ O. Zeman, Phys. Fluids A 2, 178 (1990).

${ }^{14}$ B. Aupoix, G. A. Blaisdell, W. C. Reynolds, and O. Zeman, in Studying Turbulence Using Numerical Simulation Databases, edited by P. Moin, W. Reynolds, and J. Kim (NASA-Ames/Stanford University Center for Turbulence Research, Moffett Field, CA, 1990), Vol. 3.

${ }^{15}$ C. G. Speziale and S. Sarkar, AIAA Paper No. 91-0217, 1991. 